\title{
ON DERIVATIONS OF FIELDS OF ALGEBRAIC FUNCTIONS
}

\author{
by JULIO R. BASTIDA
}

(Received 7th September 1977)

In this note, we shall prove that the transcendency degree of a finitely generated field extension is equal to a certain integer associated with a restriction mapping of spaces of derivations.

\section{Admissible mappings}

We shall assume, in this section, that all vector spaces structures under consideration are relative to a fixed field $M$.

A linear mapping of vector spaces will be called admissible when its kernel and cokernel are finite-dimensional. For every admissible linear mapping $f$, we shall put ind $(f)=\operatorname{dim}(\operatorname{Ker}(f))-\operatorname{dim}(\operatorname{Coker}(f))$, and say that ind $(f)$ is the index of $f$.

Admissible mappings and their indices are discussed in (1). We shall only require the following elementary result.

Let $X, Y$, and $Z$ be vector spaces. If $f$ and $g$ are respectively admissible linear mappings from $X$ to $Y$ and from $Y$ to $Z$, then $g \circ f$ is admissible, and ind $(g \circ f)=$ ind $(g)+\operatorname{ind}(f)$.

\section{Notation}

Given a field $R$ and an extension $M$ of $R$, the symbol $\operatorname{Der}(R, M)$ will denote the set of all derivations from $R$ to $M$, provided with its natural structure of vector space over $M$. Furthermore, if $Q$ is a subfield of $R$, the symbol $\operatorname{Der}_{Q}(R, M)$ will denote the subspace of $\operatorname{Der}(R, M)$ consisting of all $Q$-derivations from $R$ to $M$.

\section{The main result}

Throughout this section, we shall consider a field $K$, an extension $L$ of $K$, and an extension $M$ of $L$.

We shall denote by $\operatorname{res}_{K, L, M}$ the linear mapping from $\operatorname{Der}(L, M)$ to $\operatorname{Der}(K, M)$ which assigns to each derivation from $L$ to $M$ its restriction to $K$.

It is evident that $\operatorname{Ker}\left(\operatorname{res}_{K, L, M}\right)=\operatorname{Der}_{K}(L, M)$, and that $\operatorname{Im}\left(\operatorname{res}_{K, L, M}\right)$ is the subspace of $\operatorname{Der}(K, M)$ consisting of all derivations from $K$ to $M$ which are extendible to derivations from $L$ to $M$. Consequently, to say that $\operatorname{Coker}\left(\operatorname{res}_{K, L, M}\right)$ is null means that every derivation from $K$ to $M$ is extendible to a derivation from $L$ to $M$.

We are now in a position to state and prove our result. 
Theorem. If $L$ is finitely generated over $K$, then $\operatorname{res}_{K, L_{-} M}$ is admissible, and

$$
\text { ind }\left(\operatorname{res}_{K, L, M}\right)=\operatorname{tr} \text {. deg. }(L / K) \text {. }
$$

In order to prove this theorem, we shall require the following:

Lemma. The validity of the theorem follows from its validity in the particular case where $L$ is a simple extension of $K$.

Proof of the lemma. We proceed by induction on the number of generators of the intermediate field over the bottom field. The case of one generator is the particular case assumed. Now assume that $n \geqq 1$ and that the theorem is valid for the case of $n$ generators. Consider then the situation where $L=K\left(x_{1}, \ldots, x_{n}, x_{n+1}\right)$, and put $Q=K\left(x_{1}, \ldots, x_{n}\right)$. It is evident that $L=Q\left(x_{n+1}\right)$, and our assumptions then imply that res $K, Q, M$ and res $Q, L, M$ are admissible, and ind $\left(\operatorname{res}_{K, Q, M}\right)=\operatorname{tr}$. deg. $(Q / K)$ and ind $\left(\operatorname{res}_{Q, L, M}\right)=\operatorname{tr}$. deg. $(L / Q)$. Since

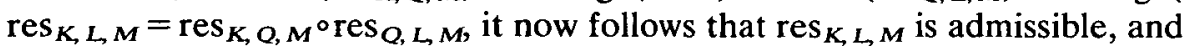

ind $\left(\operatorname{res}_{K, L, M}\right)=$ ind $\left(\operatorname{res}_{K, Q, M}\right)+$ ind $\left(\operatorname{res}_{Q, L, M}\right)=\operatorname{tr}$. deg. $(Q / K)+\operatorname{tr}$. deg. $(L / Q)$ $=$ tr. deg. $(L / K)$.

Proof of the theorem. By virtue of the lemma, we can assume that $L=K(x)$ for some $x \in L$. As the theorem is trivial when $K=L$, we shall assume that $x \notin K$.

We consider four possible cases. In discussing each of these, we shall invoke wellknown results on derivations of fields. These are presented as consequences of Theorem 39 of Chapter II of (2).

Case 1. $x$ is transcendental over $K$.

We shall apply twice Corollary 1 , page 124 of (2). First, since every derivation from $K$ to $M$ is extendible to a derivation from $L$ to $M$, we see that Coker (res $\operatorname{ros}_{K, M}$ ) is null. Also, there exists a unique $\bar{D} \in \operatorname{Der}_{K}(L, M)$ such that $\bar{D}(x)=1$; as $D=D(x) \bar{D}$ for $D \in$ $\operatorname{Der}_{K}(L, M)$, we have $\operatorname{dim}\left(\operatorname{Ker}\left(\operatorname{res}_{K, L, M}\right)\right)=\operatorname{dim}\left(\operatorname{Der}_{K}(L, M)\right)=1$. Therefore, res $\operatorname{rat}_{K, M}$ is admissible and ind $\left(\operatorname{res}_{K, L, M}\right)=1=\operatorname{tr}$. deg. $(L / K)$.

Case 2. $x$ is separably algebraic over $K$.

It is clear from Corollary 2, page 124 of (2) that $\operatorname{Ker}\left(\operatorname{res}_{K, L, M}\right)$ and $\operatorname{Coker}\left(\operatorname{res}_{K, L, M}\right)$ are null. Hence res $\operatorname{res}_{K, M}$ is admissible and ind $\left(\operatorname{res}_{K, L, M}\right)=0=\operatorname{tr}$. deg. $(L / K)$.

Case 3. $x$ is purely inseparable over $K$.

Let $p=\operatorname{char}(K)$, and let $e$ be the least integer such that $x^{p e} \in K$.

Reasoning as in case 1 , using Corollary 4, page 125 instead of Corollary 1, page 124 of (2), we deduce that $\operatorname{dim}\left(\operatorname{Ker}\left(\operatorname{res}_{K, L, M}\right)\right)=1$.

Since $x^{p e} \notin K^{p}$, it is clear from the remark in page 126 of (2) that there exists a derivation $\tilde{D}$ from $K$ to $M$ such that $\tilde{D}\left(x^{p^{e}}\right)=1$. We shall now verify that $\operatorname{Der}(K, M)=$ $M \tilde{D} \oplus \operatorname{Im}\left(\operatorname{res}_{K, L, M}\right)$, from which it will follow that Coker $\left(\operatorname{res}_{K, L, M}\right) \cong M \tilde{D}$, and hence $\operatorname{dim}\left(\right.$ Coker $\left.\left(\operatorname{res}_{K, L, M}\right)\right)=1$.

First suppose that $D \in M \tilde{D} \cap \operatorname{Im}\left(\operatorname{res}_{K, L, M}\right)$. Then $D$ is extendible to a derivation $D^{\prime}$ from $L$ to $M$, whence $D\left(x^{p e}\right)=D^{\prime}\left(x^{p e}\right)=p^{e} x^{p e-1} D^{\prime}(x)=0$; also, $D=m \tilde{D}$ for some $m \in M$, and hence $m=m \tilde{D}\left(x^{p e}\right)=D\left(x^{p e}\right)=0$. It follows that $D=0$.

Now, let $D \in \operatorname{Der}(K, M)$, and put $m=D\left(x^{p^{e}}\right)$. Then $(D-m \tilde{D})\left(x^{p^{e}}\right)=$ $D\left(x^{p^{e}}\right)-m \tilde{D}\left(x^{p e}\right)=m-m=0$. We then know from Corollary 4, page 125 of $(2)$ that this 
implies the extendibility of $D-m D$ to a derivation from $L$ to $M$, and hence that $D-m \tilde{D} \in \operatorname{Im}\left(\operatorname{res}_{K, L, M}\right)$. It then follows that

$$
D=m \tilde{D}+(D-m \tilde{D}) \in M \tilde{D}+\operatorname{Im}\left(\operatorname{res}_{K, L, M}\right) \text {. }
$$

Thus, $\operatorname{res}_{K, L, M}$ is admissible and ind $\left(\operatorname{res}_{K, L, M}\right)=0=$ tr. deg. $(L / K)$.

Case 4. $x$ is algebraic and inseparable over $K$.

Let $S$ denote the separable closure of $K$ in $L$. Then $L=S(x), x \notin S$, and $x$ is purely inseparable over $S$; it follows from the third case, in particular, that res $S, L, M$ is admissible and ind $\left(\operatorname{res}_{S, L, M}\right)=\operatorname{tr}$. deg. $(L / S)=0$. Since $S$ is separably algebraic over $K$, we deduce from the second case and the theorem of the primitive element that $\operatorname{res}_{K, S, M}$ is admissible and ind $\left(\operatorname{res}_{K, S, M}\right)=\operatorname{tr}$. deg. $(S / K)=0$.

As res ${ }_{K, L, M}=\operatorname{res}_{K, L, M}{ }^{\circ} \operatorname{res}_{S, L, M}$, we now conclude that res ${ }_{K, L, M}$ is admissible and ind $\left(\operatorname{res}_{K, L, M}\right)=$ ind $\left(\operatorname{res}_{K, S, M}\right)+$ ind $\left(\operatorname{res}_{S, L, M}\right)=0=$ tr. deg. $(L / K)$.

Remark. In Chapter II of (2), Theorems 40, 41, and 42 study the relation between separability and properties of derivations. It is easily seen that Theorems 40 and 41 are immediate consequences of our result and Theorem 42.

\section{REFERENCES}

(1) R. S. PALAIS, Seminar on the Atiyah-Singer Index Theorem (Annals of Mathematics Studies, No. 37, Princeton, 1965).

(2) O. ZARISKI and P. SAMUEL, Commutative Algebra, Vol. I (D. van Nostrand Co., Inc., Princeton, 1958).

FLORIDA ATLANTIC UNIVERSITY

BOCA RATON, FLORIDA 33432 